\title{
The Effect of Dividing the Magnets on the Calculation of the Recycler Dynamic Aperture.
}

\author{
Norman Gelfand
}

May 4, 2001

\section{Introduction}

The modeling of a lattice and the calculation of the dynamic aperture, are exercises fraught with uncertainty and possible error. If we want an accurate value for the dynamic aperture, we must start with an accurate model for the lattice. This model should incorporate the misalignments, including the rolls, of the lattice elements, as well as the error fields in the magnets. These quantities are not always well known.

It has been known for sometime that long term tracking must be symplectic. (Reference 1). This means that either we utilize a code like MARYLIE (Reference 2) which employs a Lie algebraic approach, or a code that employs zero length kicks for the non-linear parts of the magnetic fields. ${ }^{1}$

Calculation of the dynamic aperture of the Recycler is particularly difficult because of the large intrinsic sextupole built into gradient magnets.

A recent attempt (Reference 3) to calculate the dynamic aperture for the Recycler Ring has used $M A D$. Originally the calculation was done with the magnets treated, as they exist, as single objects. The result was a dynamic aperture of $3.0 \sigma .^{2}$ The calculation was redone with the magnets split into 16 segments and the result was a dynamic aperture of $9.5 \sigma$. This report is an attempt to understand the reason for the difference in these two calculations.

\footnotetext{
${ }^{1}$ Examples of such codes are TEVLAT and TEAPOT. In TEAPOT non-zero length linear elements are also replaced, with zero length kicks and drifts.

${ }^{2}$ The $\sigma$ of the beam, at any point in the lattice is calculated from the emittance $\epsilon$ of the beam and the value of the lattice function $\beta . \sigma=\sqrt{(\epsilon \cdot \beta)}$
} 


\section{The $M A D$ Calculation}

The Recycler Ring is constructed from combined function rectangular magnets. The field in most of these magnets contains a sextupole component in addition to the dipole and quadrupole components.

The lattice description used, both here and in Reference 3, is contained in a file, RRv20base.mad. In this file the lattice bend magnets, are described as sector magnets, rather than as the rectangular magnets which are actually found in the tunnel. ${ }^{3}$

The dipole fields in the lattice file are the nominal design values. The multipole field components for each magnet were measured at the Magnet Test Facility(MTF). The variation in the dipole field was incorporated into the $M A D$ tracking via an error field. It results in a small closed orbit distortion.

The lattice description does however, contain the values measured at MTF for the quadrupole and sextupole moments for each individual magnet. Thus the magnet to magnet variation of these components is incorporated into the lattice description rather than as multipole kicks or error fields. The measured multipoles higher than the sextupole for each magnet, were incorporated into the $M A D$ tracking via multipole kicks at the ends of each magnet.

The dynamic aperture was calculated by tracking in $M A D$ for $10^{5}$ turns. The tracking was begun with different values for the emittance in one plane. The maximum emittance in the other transverse plane was determined by the stability of the particle. No physical aperture was imposed on the particles during the tracking. The dynamic aperture was calculated to be $3.0 \sigma$.

In an attempt to understand, and perhaps improve on the original result, the combined function magnets were divided into 16 segments and the tracking was repeated. A significantly greater dynamic aperture, $9.5 \sigma$, was calculated.

\section{Understanding these Results- A Tracking Analysis}

The following analysis will consist of three parts:

\footnotetext{
${ }^{3}$ I assume that this was done to resolve a perceived problem of how to handle, within $M A D$, the edge focusing when the magnets were split. This problem does not exist in TEVLAT.
} 
1. TEVLAT Tracking to Calculate the Dynamic Aperture.

TEVLAT was used to calculate the dynamic aperture. In TEVLAT one can specify the number of pieces into which a magnet will be divided during the calculation. The results for different values for the number of splits can then be compared.

2. Tracing through a single magnet.

Code was written to study the change in the final coordinates and slopes when a single magnet was divided into multiple pieces. For the sextupole field this was done for both the TRANSPORT (Reference 4) maps as used in $M A D$ and for kicks, as in TEVLAT.

\section{Multipass Tracking.}

Special code was written to allow multipass tracking of a particle through a single magnet. The transform through the magnet was done with both the TRANSPORT maps and with kicks ala TEVLAT.

\section{Dynamic Aperture As Calculated in TEVLAT}

TEVLAT is a tracking code developed for use with the Tevatron (Reference $5)$. It has been adapted for use with other lattices. Linear elements are described by transfer matrices, and non-linearities are described by zero length kicks. The tracking is therefore symplectic.

In the case of a lattice element which is linear, but with a small nonlinearity (the high order multipoles or HOM), the magnet is divided into a number of pieces, typically two. The pieces are treated within TEVLAT as linear elements while the non-linearities are lumped into a kick between the pieces.

To handle the body sextupole of the long gradient magnets in the Recycler a different algorithm was used. Sextupole kicks are inserted at the entrance to, in the middle of, and at the exit from the magnet. Between the kicks the magnet is treated as a linear element. The tracking is still symplectic since the non-linear sextupoles are approximated with zero length kicks.

A comparison of the TEVLAT computation of the chromaticity with the $M A D$ computation gives good, but not perfect agreement.

The natural chromaticity of this lattice, that is the chromaticity without 
any sextupole correction, is $\zeta_{x} \approx-30$ and $\zeta_{y} \approx+21 .{ }^{4}$ With the sextupoles turned on $M A D$ computes as the chromaticity

$\zeta_{x}=-2.03$ and $\zeta_{y}=-2.72$.

The corresponding values from TEVLAT are

$\zeta_{x}=-3.22$ and $\zeta_{y}=-3.03$.

There is substantial agreement for the change in the chromaticitiy in the two calculations.

TEVLAT was used to compute the dynamic aperture only for the case where $\epsilon_{x}=\epsilon_{y} \cdot{ }^{5}$ In order to compare the TEVLAT results with those reported in Reference 3 tracking was done for $10^{5}$ turns.

There are two other differences, other than the differences in the programs, between the calculations in TEVLAT and in MAD. First the dipole errors are not incorporated into the TEVLAT calculation, thus there are no closed orbit distortions. The measured dipole errors are small and therefore should have only a small effect on the dynamic aperture. The errors should not effect this study of the effect of splitting the magnets.

The second difference is that in the $M A D$ calculations the multipole kicks from the magnet errors are included before and after the magnet. In TEVLAT the kicks are calculated and applied at the middle of each magnet. Because the multipoles are at the ends of the magnets in $M A D$, splitting the magnets has no effect on the computed multipole kicks. In TEVLAT dividing the magnet distributes the multipole kicks along the length of the magnet, perhaps a more realistic approximation. The location at which we apply the multipole kicks should not be very important in studying the effect of splitting the magnets on the dynamic aperture.

The results of the TEVLAT calculations are shown in Table I along with the results from Reference 3 . The dynamic aperture is given in terms of the normalized admittance ${ }^{6}$, as well as in terms of the beam $\sigma$ assuming that the $95 \%$ normalized emittance of the beam is $18 \pi m m-m r$.

\footnotetext{
${ }^{4} M A D$ and TEVLAT give the same result.

${ }^{5}$ This was done solely to save computing time.

${ }^{6}$ It is not clear how the emittances given in Reference 3 are calculated.
} 
Table I Calculated Dynamic Aperture

\begin{tabular}{|l|l|l|l|}
\hline $\begin{array}{l}\text { \# of } \\
\text { Pieces }\end{array}$ & $\begin{array}{l}\text { Max } \epsilon_{x}=\epsilon_{y} \\
(m m-m r)\end{array}$ & DA $(\sigma)$ & MAD $D^{3}$ \\
\hline 1 & 216.75 & 12.0 & 3.0 \\
\hline 2 & 192.0 & 11.3 & \\
\hline 4 & 192.0 & 11.3 & \\
\hline 8 & 216.75 & 12.0 & \\
\hline 16 & 216.75 & 12.0 & 9.5 \\
\hline
\end{tabular}

Two points are worth noting. The calculations done with TEVLAT do not show any significant dependence on the number of times the magnets are split, a result at variance with the $M A D$ calculations. The second point is that the dynamic aperture as calculated by TEVLAT is $2.5 \sigma$ larger than the $M A D$ calculation.

\section{Discussion}

As already mentioned the magnets in the Recycler are gradient magnets and incorporate a sextupole component for chromaticity correction. In $M A D$ this non-linearity is incorporated in the second order TRANSPORT map $T_{i j k}$. In the MAD Physical Methods Manual we find the following comment:

"Note that the TRANSPORT map tracking is not symplectic; for long term tracking it most certainly causes spurious blow up or shrinkage." (emphasis added)

The results reported in Reference 3 were done in $M A D$ using the TRANSPORT maps. The quoted results are consistent with the idea that during the tracking the emittance blows up because the tracking is non-symplectic, i.e. that it is a spurious blow up, not due to the underlying dynamics. The dynamic aperture calculated is therefore, smaller than the actual dynamic aperture.

Dividing the magnets into a number of pieces means that each piece has a TRANSPORT map with smaller elements than the original map. We can view this process, dividing the magnets, as replacing the finite length elements, in the limit, with zero length kicks. Zero length kicks represent a symplectic map and so as we increase the number of pieces the maps are closer to being symplectic. In order to observe the blow up due to the nonsymplectic nature of the map we would need to track for more turns as we 
increase the number of pieces. Failing that we could observe a larger dynamic aperture with the magnets split. That of course is what is observed.

\section{Tracing Through a Single Magnet}

A particle has been traced through a single Recycler magnet in order to see the change in the final coordinates, as a magnet is divided in a different number of pieces. Both the TRANSPORT maps and TEVLAT algorithm were used. The magnet chosen is at Recycler location 111a. This magnet has a large sextupole component and studying it therefore might help us understand the effects of the non-linearities on the tracking.

A particle with an amplitude correspond to a particle with a normalized emittance of $200 \pi m m-m r$ was traced through the magnet. The particle launched from a number of locations on the ellipse in phase space corresponding to this emittance.

Figure 1 shows the change in the final coordinate of a particle, as calculated using the TRANSPORT second order maps when the magnet is divided into a different number of segments (the initial angle in phase space is also varied). We observe that the final coordinate is insensitive to the number of pieces into which we divide the magnet, never being more the $0.05 \mu \mathrm{m}$ from the case where the magnet is not divided. Figure 2 shows the variation in the final slope of the particle. The maximum change is less than $0.05 \mu \mathrm{r}$ Thus, it is fair to say that, dividing a magnet in $M A D$ makes only a tiny change in the exit coordinates for a given initial emittance.

In the method where the non-linearities are handled as kicks, as in TEVLAT, the comparison is made with the case where the magnet is split into 32 pieces. The result (Figure 3) show that as the number of splits is changed, the variation is larger than with the TRANSPORT maps. The change only exceeds $\approx 3 \mu \mathrm{m}$ when the magnet is not split. When the magnet is split into two pieces the difference, from the case where the magnet is divided in to 32 pieces is less than $\approx 1 \mu \mathrm{m}$. Similar results are obtained when looking at the variation in the slope (Figure 4), as the magnet is divided in to different numbers of pieces.

Finally comparing the results from the TRANSPORT maps with the kick calculations, when the number of splits is two or more the difference in the

calculations is less than $1 \mu \mathrm{m}$ for the position or $1 \mu r$ for the slope. (Figure 5, Figure 6.) 
From these calculations we can conclude that:

1. Using the TRANSPORT map in $M A D$ for this magnet there is at most a minute change in the final coordinate of a particle when the element is divided;

2. Using the algorithm used in TEVLAT the dependence of the exit coordinate on the number of pieces into which the magnet is divided, is small when the number of pieces is two or more.

3. The difference between the two calculations for the final coordinate is also small when the magnet is divided into two or more pieces for the TEVLAT calculation.

\section{Multipass Tracking}

To demonstrate the effect of the non-symplectic TRANSPORT map used in $M A D$ on long-term tracking a simple model was constructed. ${ }^{7}$ The particle is tracked through a single magnet using the TRANSPORT map or with kicks. The turn is then completed using a simple linear transfer matrix, generated from the values of the linear lattice functions at the start and end of the magnet. The phase of this matrix was chosen so that the fractional tune in each plane is 0.4, the nominal fractional tune of the Recycler.

This tracking can not and should not be regarded as a calculation of the dynamic aperture of the Recycler but should gives us some insight into the $M A D$ tracking.

The particle is tracked for up to $10^{6}$ turns but tracking is terminated if the particle's coordinate goes outside of an imposed aperture of $35 \mathrm{~mm}$. In order to characterize the behavior of the particle the average and the $\sigma$ of the position of the particle were computed for every $10^{4}$ turns. $^{8}$ The average position is always zero within the expected variance. Using the TRANSPORT map, as in $M A D$, the $\sigma$ of the beam shows a continuous increase in size as the

\footnotetext{
${ }^{7}$ This method is an adaptation of the work of Leo Michelotti in his paper Perturbation Theory and the Single Sextupole (Reference 6).

${ }^{8}$ The $\sigma$ is used to characterize the tracking rather than the linear invariant $\epsilon$ because the system is not linear and because of the difference between the lattice functions calculated using TEVLAT and the TRANSPORT map.
} 
number of turns increases. After about $250 \mathrm{~K}$ turns beam position exceeds the imposed aperture. (Figure 7).

Tracking with the sextupole in the magnet approximated with kicks (with TEVLAT), which is a symplectic mapping, there is no corresponding divergence in the $\sigma$ of the particle's coordinate. ${ }^{9}$ This result helps us understand why the dynamic aperture calculated using a kick code can be greater than the dynamic aperture calculated by the non-symplectic TRANSPORT maps.

The tracking was repeated with the magnet divided into up to 16 segments. The results are shown in Figure 8 and Figure 9. In this case the behavior of the beam $\sigma$, when tracking with the TRANSPORT maps depends on the number of segments into which the magnet is divided. With two segments the beam $\sigma$ increases as we track but with four or more segments the beam $\sigma$ decreases the longer we track. Thus, the non-symplectic tracking can result in either an increased or decreased calculated dynamic aperture depending on the details of how the magnets are represented. The scale, i.e. the number of turns, for the growth or shrinkage depends on the number of segments, increasing as the number of segments increases. This is what would be expected if the effect on the beam $\sigma$ is due to the nonsymplectic nature of the TRANSPORT maps.

As stated above this simple model should not be expected to give the behavior for the tracking of the full Recycler lattice as the segmentation is increased. It does however indicate the great caution that must be exercised before dynamic aperture calculations with non-symplectic transformations are used.

\section{Conclusion}

It is to be hoped that the calculation reported above again convincingly demonstrates that tracking with the TRANSPORT maps in MAD cannot be relied upon to give a realistic value for the dynamic aperture. That is because the maps are not symplectic. Even dividing the magnet into a large, but finite, number of segments not does guarantee a reliable answer. The calculated dynamic aperture may be realistic but I, at least, do not know how to demonstrate that it is. As noted earlier, this is not a new observation, but was known by the authors of $M A D$ among others.

\footnotetext{
${ }^{9}$ This statement depends on the tune. Near a resonance the beam $\sigma$ diverges.
} 
It is hoped that the dynamic aperture obtained in the symplectic tracking code TEVLAT is a realistic approximation to the actually dynamic aperture.

\section{References.}

1. Lectures on Nonlinear Dynamics,Alex J. Dragt;Physics of High Energy Accelerators (Fermilab Summer School, 1981) (AIP Conference Proceedings No. 87) edited by R. A. Carrigan, F. R. Huson, M. Month

2. MARYLIE 3.0 - A Program for Nonlinear Analysis of Accelerator and Beamline Lattices A.J. Dragt et al., IEEE Trans. Nucl Sci NS-32 2311

3. Dynamic Aperture in the Recycler Ring, Meiqin Xiao and Tanaji Sen.

4. Third Order TRANSPORT with MAD Input D.C. Carey, K.L. Brown, F. Rothacker,SLAC Report 530

5. TEVLAT private communication, A. Russell.

6. Perturbation Theory and the Single Sextupole,Leo Michelotti; The Physics of Phase Space edited by Y. S. Kim and W. W. Zachary (Springer-Verlag 1987 
Change in Final Position; Vary starting phase, split

cfg111a-Transport Map

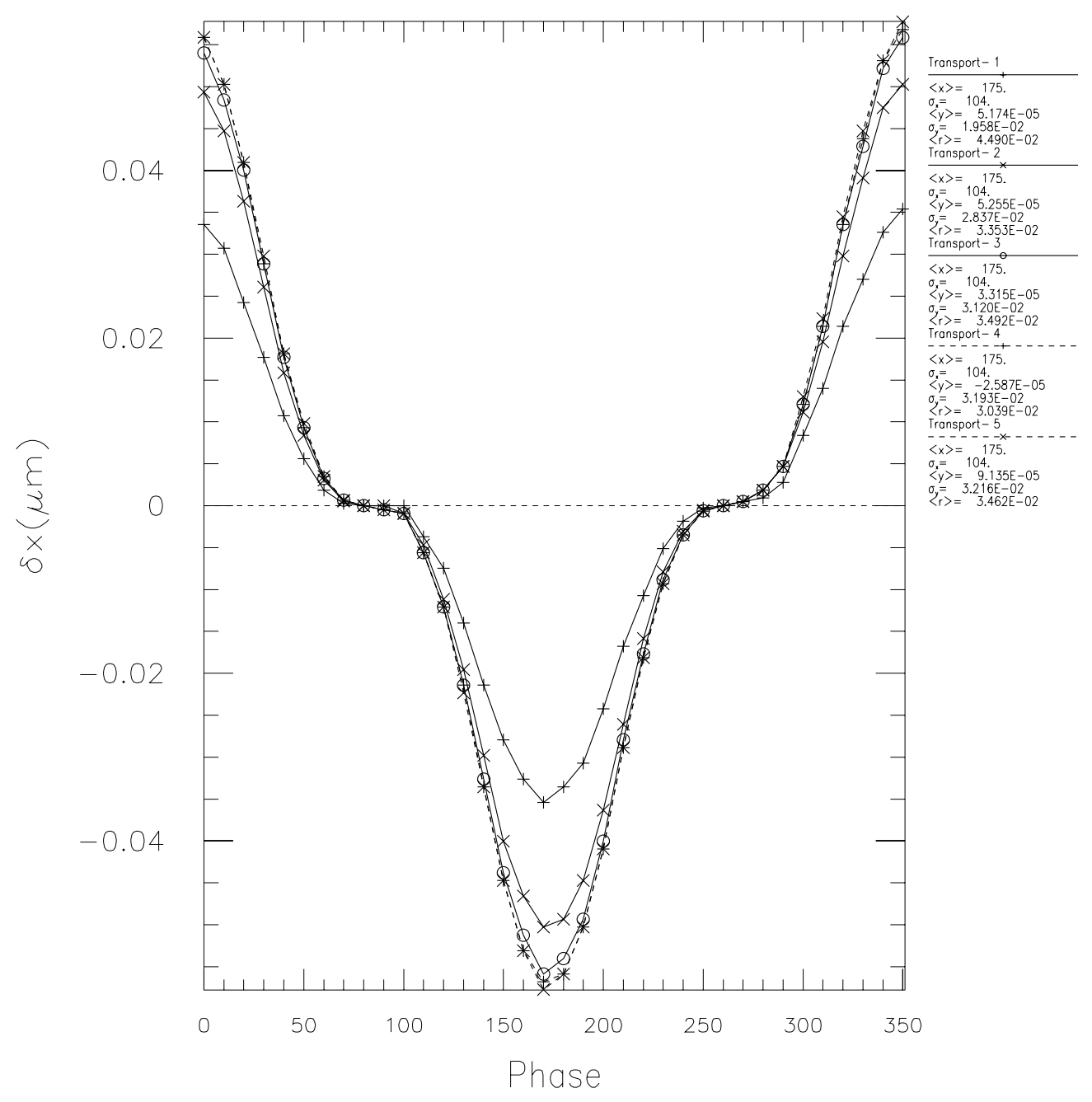

Figure 1: Comparison of the Change in Position- TRANSPORT Map. 


\section{Change in Final Slope; Vary starting phase,split cfg111a-Transport Map}

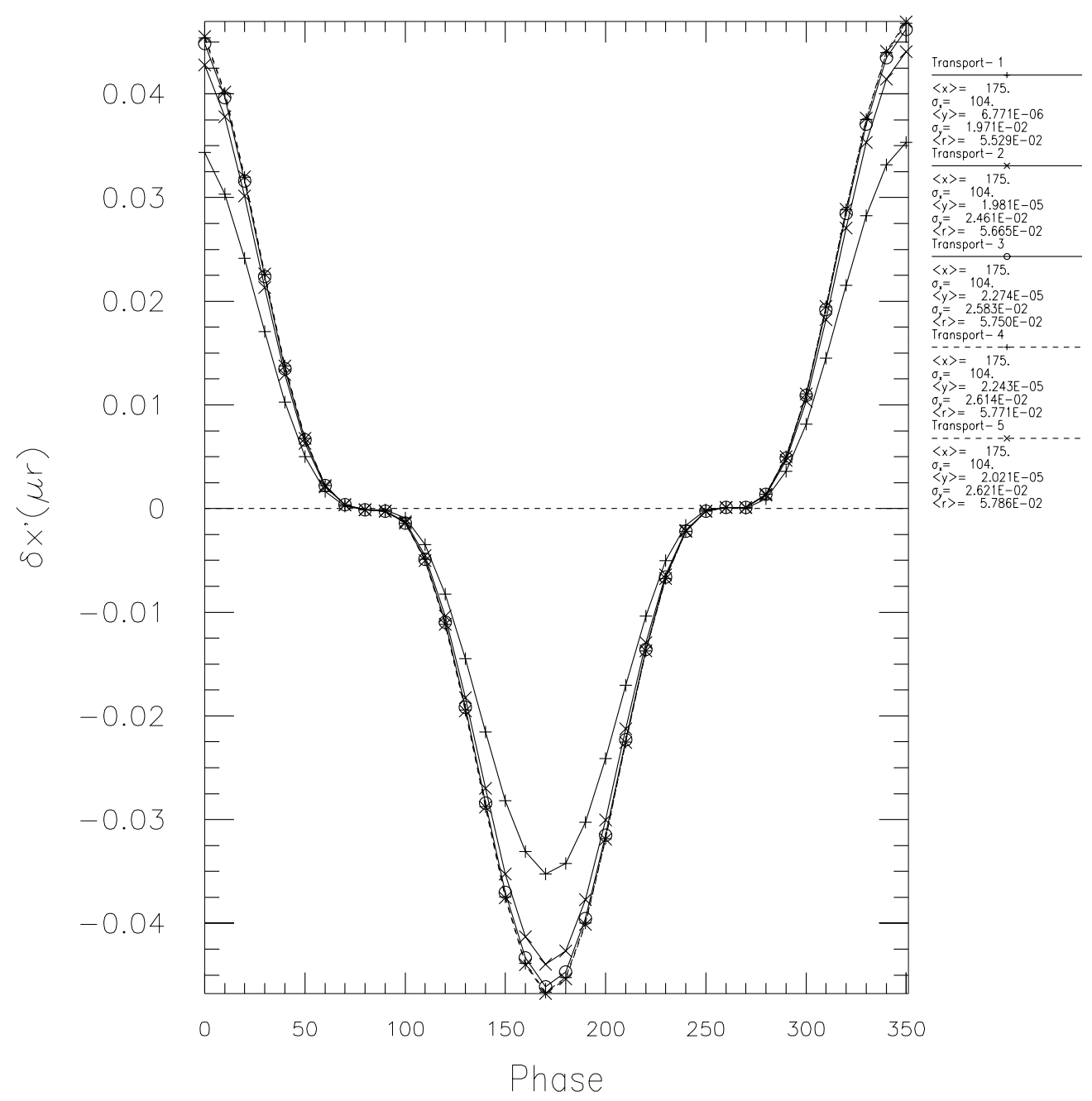

Figure 2: Comparison of the Change in Slope- TRANSPORT Map. 


\section{Change in Final Coordinate; Vary starting phase,split \\ cfg111a-Kicks}

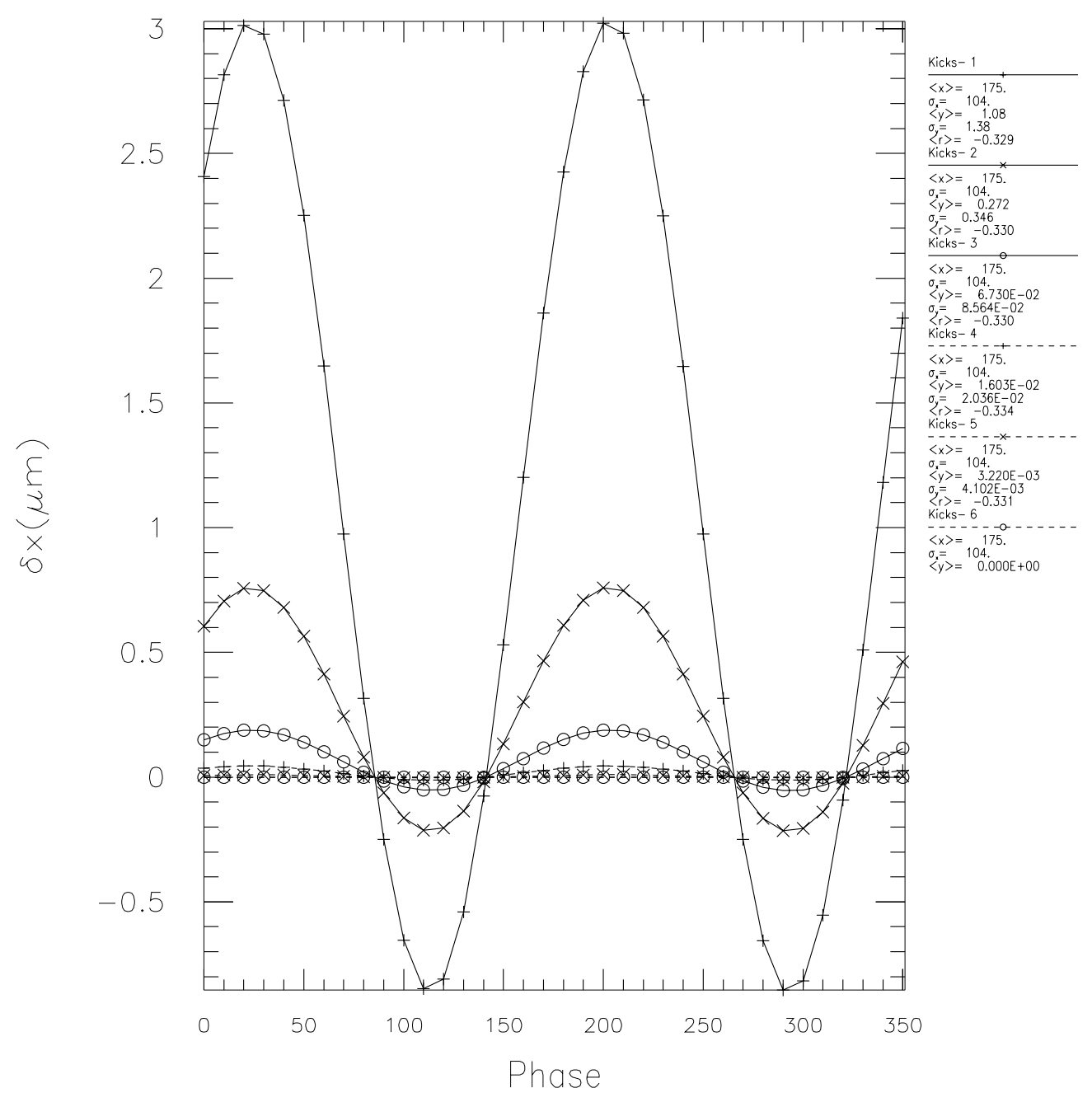

Figure 3: Comparison of the Change in Position- Kicks. 


\section{Change in Final Slope; Vary starting phase, split cfg111a-Kicks}

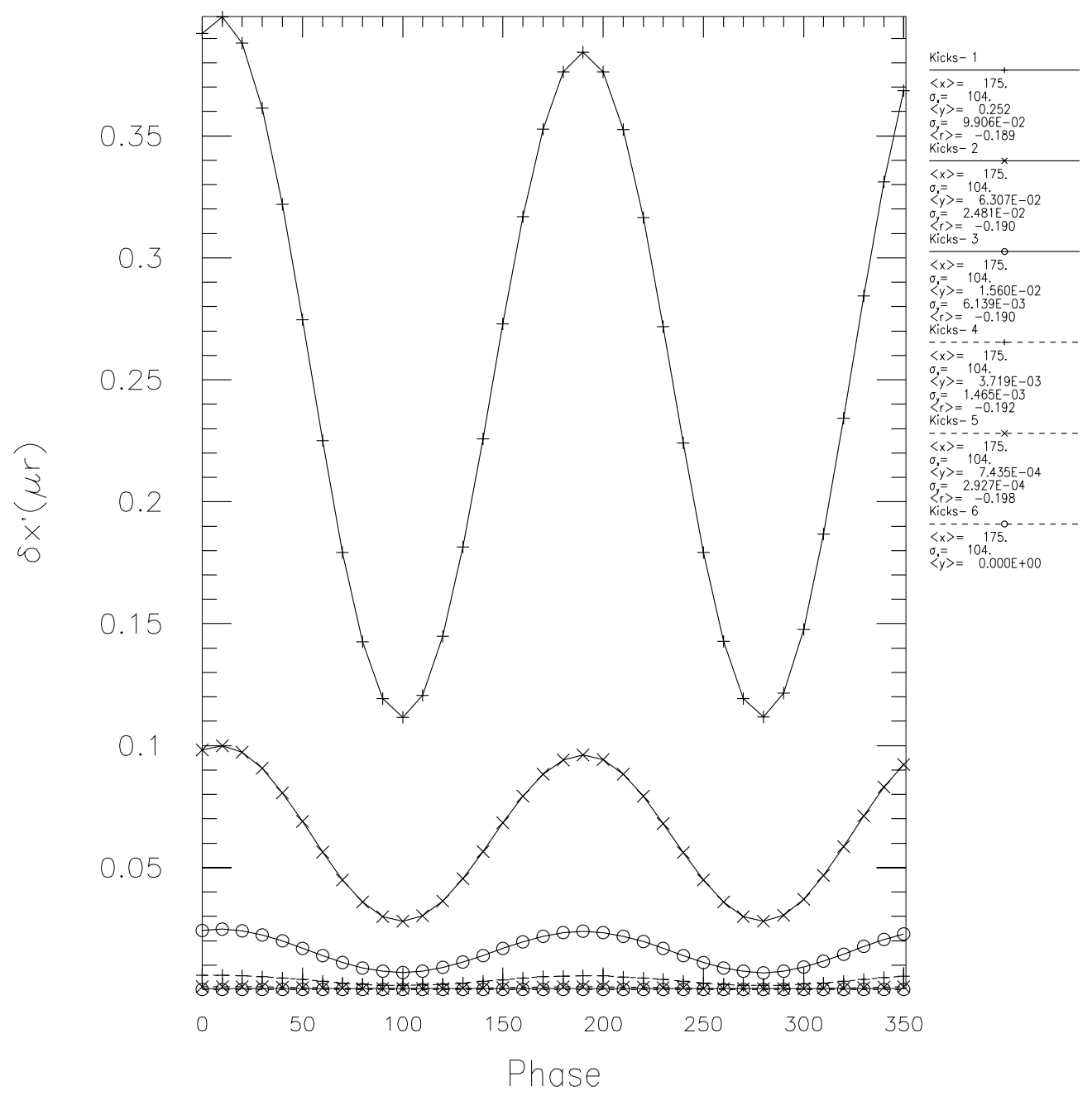

Figure 4: Comparison of the Change in Slope - Kicks. 


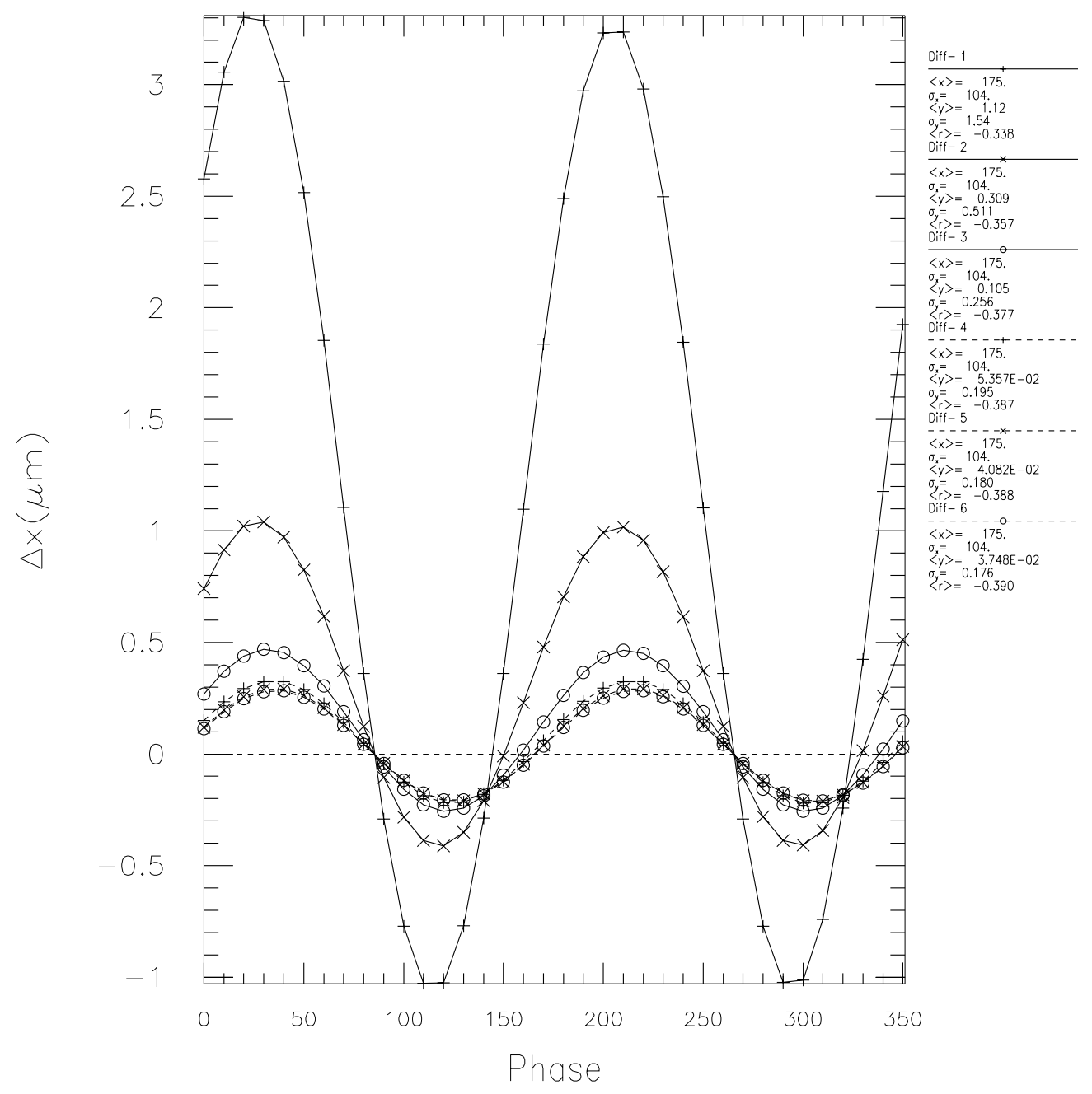

Figure 5: Comparison of the Final Position; TRANSPORT Maps and Kicks. 


\section{Difference in Final Slope; Vary starting phase,split}

cfg111a Kicks - Transport

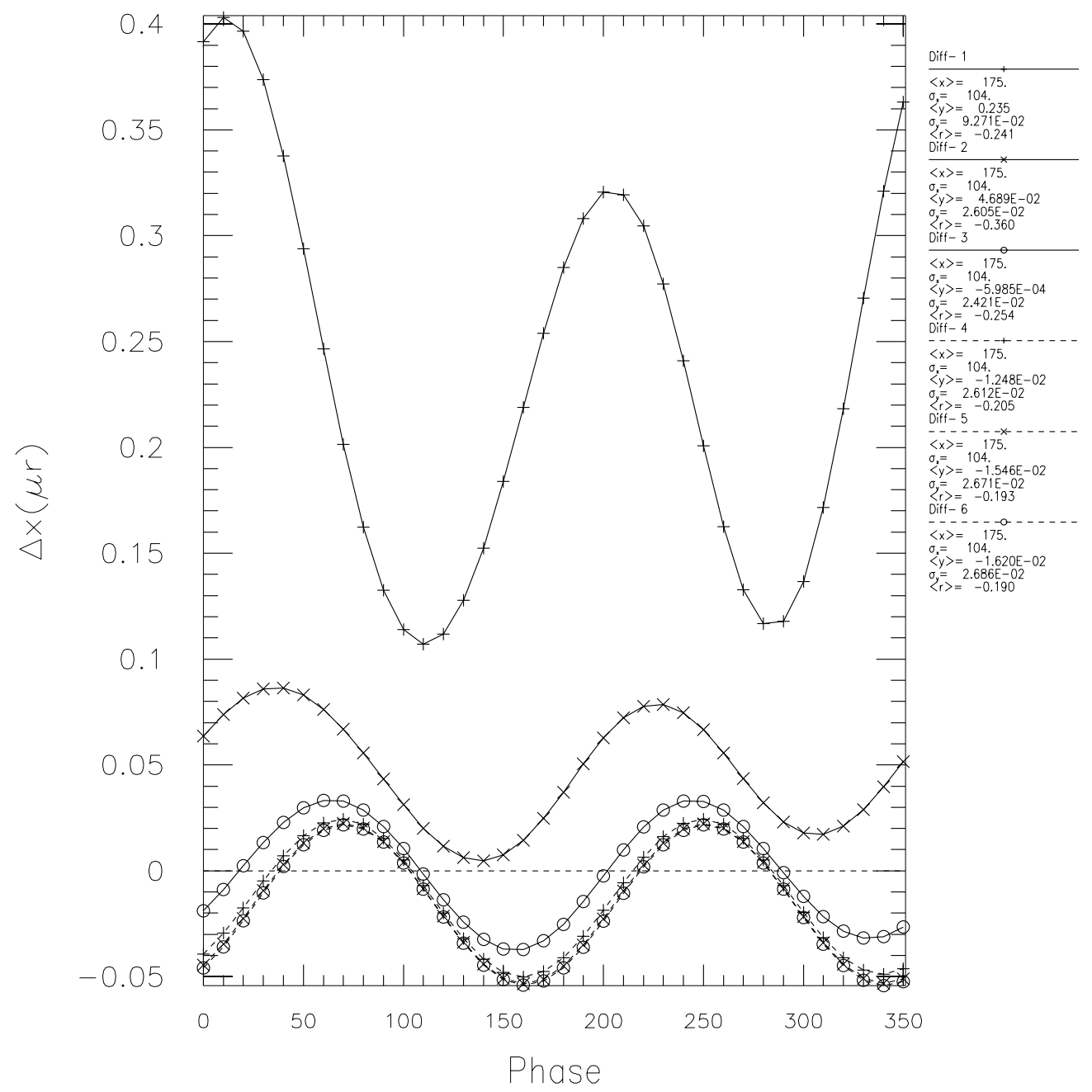

Figure 6: Comparison of the Final Slope; TRANSPORT Maps and Kicks. 


\section{Growth of Beam o vs Turn}

Rms of the beam for 10000 turns. Magnet split into 32 pieces.

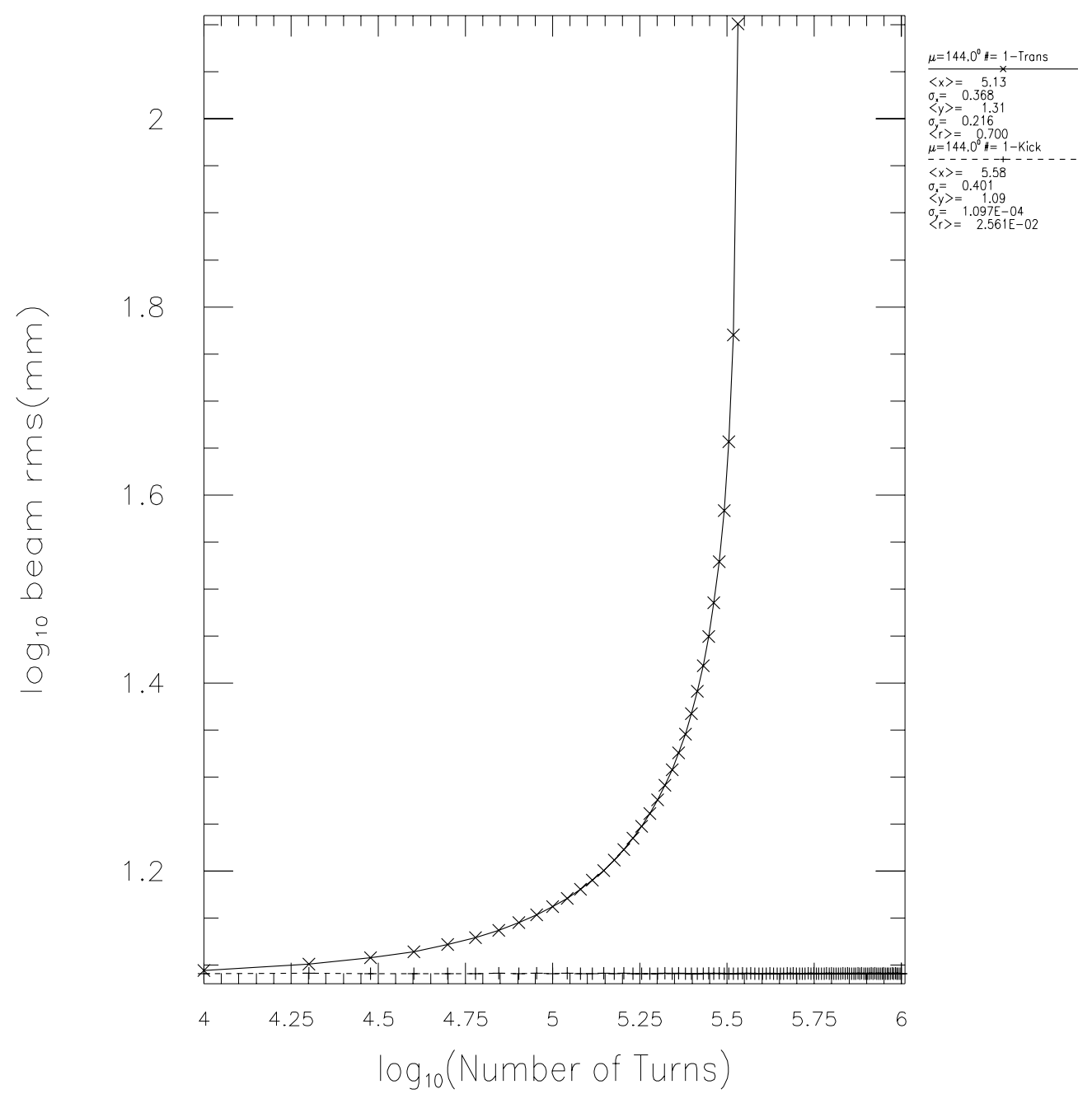

Figure 7: Beam $\sigma$ vs. Turn Number: TRANSPORT Map and Kicks. 
Rms of the beam for 10000 turns. Magnet split into 32 pieces.

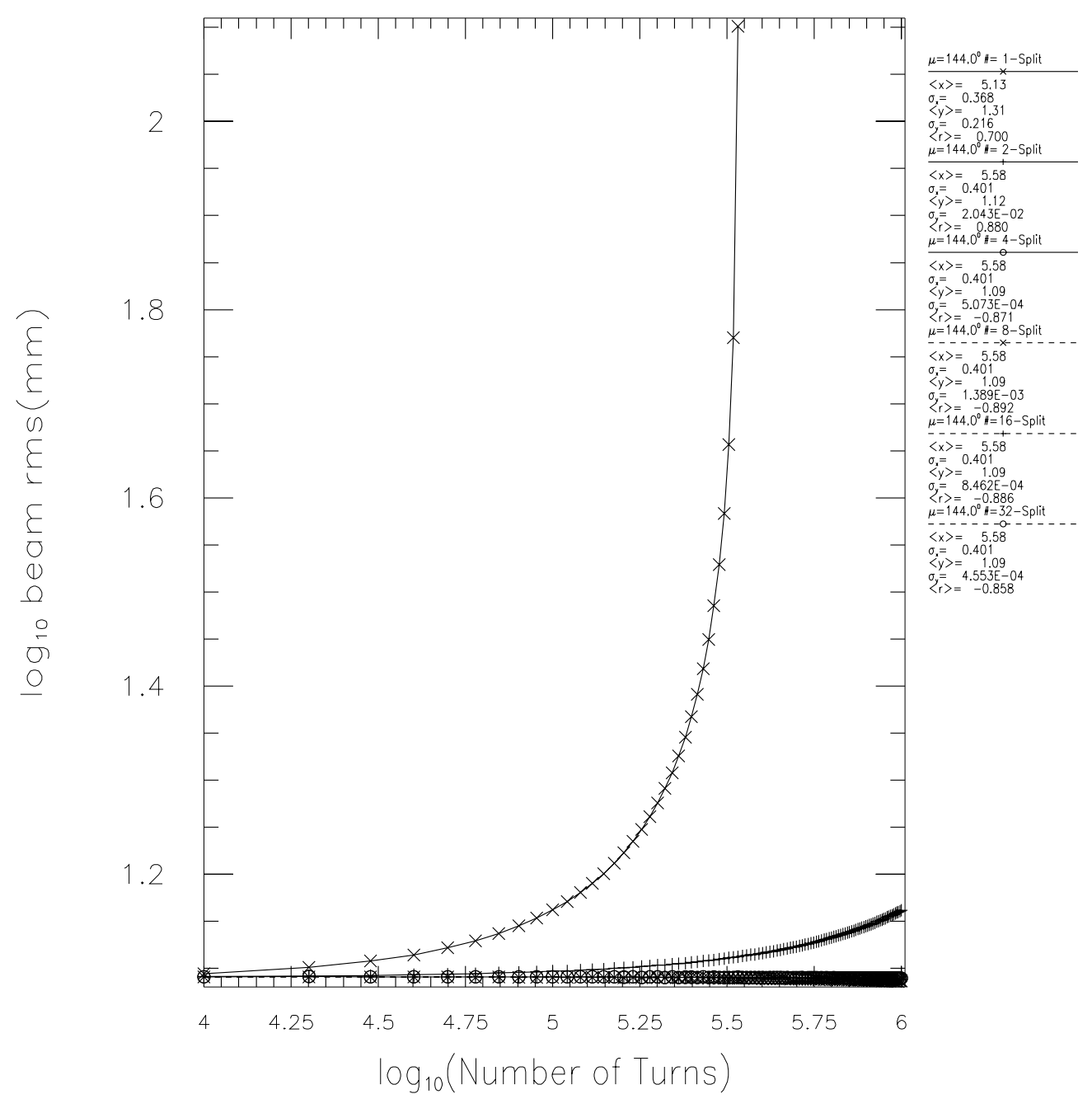

Figure 8: Beam $\sigma$ vs. Turn Number: TRANSPORT Maps. 


\section{Growth of Beam o vs Turn}

Rms of the beam for 10000 turns. Magnet split into 32 pieces.

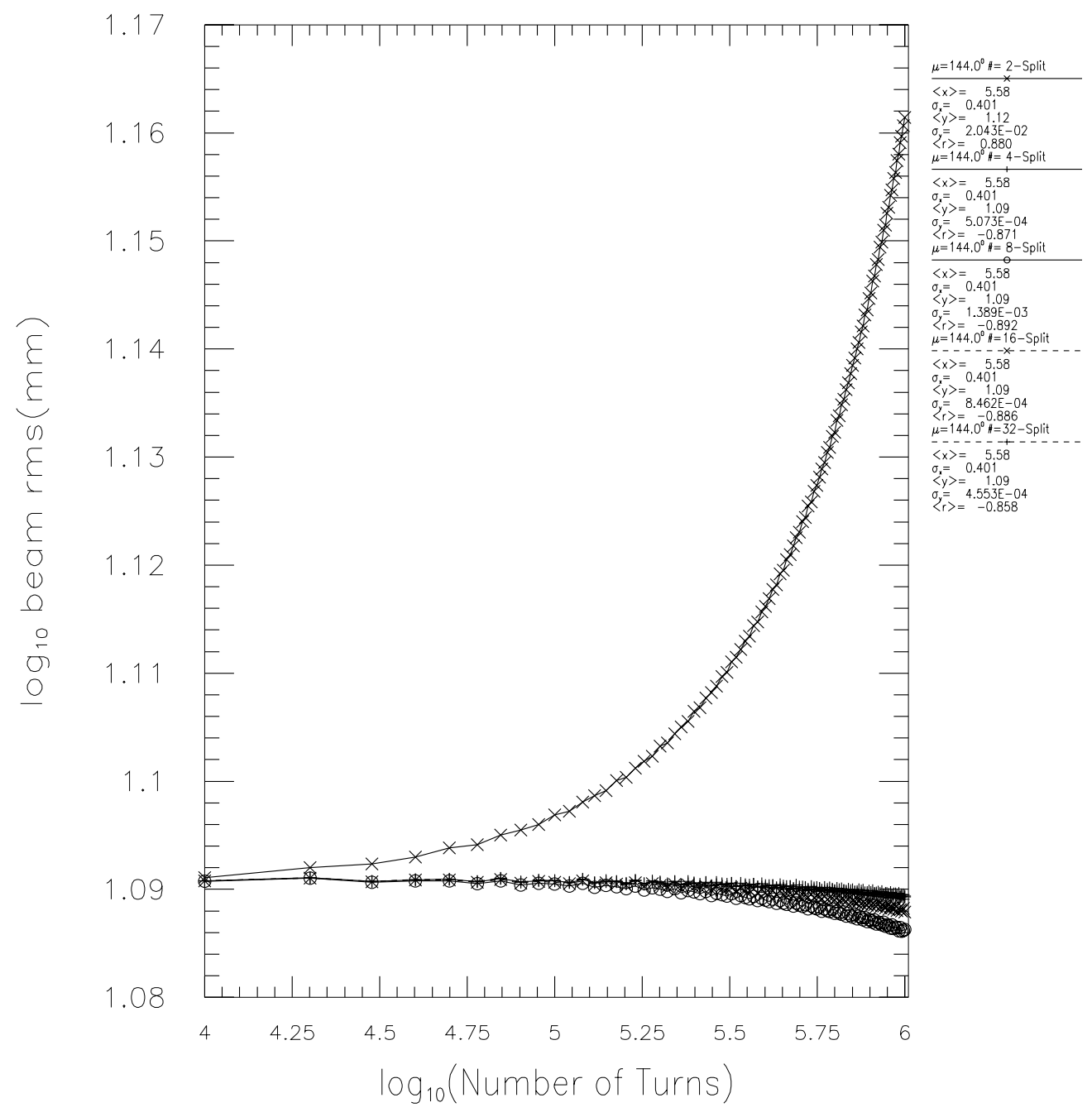

Figure 9: Beam $\sigma$ vs. Turn Number: TRANSPORT Map. Number of Segments $>1$. 